\title{
MULTICOMMODITY NETWORK FLOWS WITH NONCONVEX ARC COSTS
}

\author{
Philippe Mahey ${ }^{1}$ and Mauricio C. de Souza ${ }^{2 *}$ \\ Received May 30, 2017 / Accepted December 1, 2017
}

\begin{abstract}
We present a survey on nonconvex models and algorithms for multicommodity network design problems. We put in perspective the alternative modelling of these problems, traditionally represented by mixed-integer linear programs, by separable nonconvex arc cost functions. We show in particular that some problems take profit of a continuous modelling and explore the case of capacity expansion of communications networks.
\end{abstract}

Keywords: network design, capacity and flow assignment problem, capacity expansion.

\section{INTRODUCTION}

Multicommodity flow network optimisation problems have been widely studied and surveyed, mostly in the linear case (see [1] among others) and to some extent in the nonlinear convex case (see [77]). Most applications are still very challenging in the Network Design domain for many practitioners in different fields like transportation, communications or energy systems (see [7]).

We will focus here on the nonconvex nature of the cost function for general continuous multicommodity flows and this will include purely combinatorial problems like the pure concave cost network loading problem known to be NP-hard. To be more precise, we will consider the following model defined on a digraph $G=(\mathcal{V}, \mathcal{F})$ with a set $\mathcal{K}$ of commodities sending a fixed quantity of flow $b_{k}$ between pairs of origins and destinations $\left(s_{k}, t_{k}\right), k \in \mathcal{K}$ :

$$
\begin{aligned}
\text { Minimise } & \sum_{e \in \mathcal{F}} f_{e}\left(x_{e}\right) \\
(\mathrm{MCF}) \text { subject to } & x_{e}-\sum_{k} x_{e}^{k}=0, \forall e \in \mathcal{E} \\
& x^{k} \in \mathcal{F}_{k}, \forall k \in \mathcal{K}
\end{aligned}
$$

where $\mathcal{F}_{k}$ is the set of feasible $k$-flows:

$$
\mathcal{F}_{k}=\left\{x^{k} \mid A x^{k}=b^{k}, x^{k} \geq 0\right\}
$$

*Corresponding author.

${ }^{1}$ LIMOS-CNRS and ISIMA, Université de Clermont Auvergne, France. E-mail: philippe.mahey@isima.fr

${ }^{2}$ DEP, Universidade Federal de Minas Gerais, Brazil. E-mail: mauricio.souza@ dep.ufmg.br 
the matrix $A$ defining the arc-node incidence matrix of graph $\mathcal{G}$. Observe that the objective function is a separable arc cost function of the total flow $x_{e}=\sum_{k} x_{e}^{k}$ using arc $e \in \mathcal{E}$. In some cases, additional arc costs depending separately on each commodity must be introduced, but we will not give any special insight to them as they do not induce any notable additional difficulties in the numerical treatment of these models.

We point out that this model includes the capacitated case as the capacity constraints can be embedded in the arc cost functions $f_{e}$ which are supposed to be only piecewise smooth with values in the extended real line $\mathbb{R} \cup\{+\infty\}$. Then it can also tackle the case of discrete decisions at the condition that these are defined arcwise. In particular, we will study multicommodity flow network problems with piecewise convex arc costs which appear in the modelling of the Capacity and Flow Assignment (CFA) problems for Network Design of general data networks. On the other hand, we will not survey (unless some algorithmic tool discussed later will need to refer to it) topological constraints on the graph like path constraints or connectivity constraints.

As a basic case, the fixed cost loading problem will be modelled by the step function

$$
f_{e}\left(x_{e}\right)=\left\{\begin{array}{cc}
0 & \text { if } x_{e}=0 \\
F_{e} & \text { if } x_{e}>0
\end{array}\right.
$$

Fixed-cost as well as general concave-cost network flow problems have been largely studied since the early results of Tuy [89]. Most of these contributions, well reported in Pardalos and Rosen's survey [79], focussed on Branch-and-Bound like approaches applied to single-commodity or transshipment models. Further enhancements have improved these techniques (see [14]) and adhoc software have been produced to solve large classes of Global Optimisation problems (see $[51,57])$. These algorithmic schemes can apply too to a large class of integer flow network design problems that we will not survey here (see [11]).

The concave-cost multicommodity flow problem is much less studied in the literature even if constructive surveys have been published in the nineties (see [7, 72]). Most original approaches have faced the necessity to decompose w.r.t. commodities which led to Lagrangian relaxation and Branch-and-Price strategies. We will present in Section 3 the basic references that established the most noticeable results and algorithmic recent contributions on the fixed-charge and concave-cost Network Design problem. From uncapacitated to multiple facilities models, we will observe the importance of both polyhedral study and Benders decomposition in the literature.

The situation which will be focussed in the last section is the network design problem where routes and capacities have to be simultaneously assigned to meet a given multicommodity demand of traffic. Routing corresponds in general to convex arc costs (average delay, congestion measure, QoS...) and is usually modelled with continuous flow variables associated with each commodity unless additional constraints are present like unsplittable routing for example. On the other hand, capacity assignment has been modelled by integer decision variables as the choice is in practice modular with a finite number of available capacities for each arc. So the joint Capacity and Flow Assignment problem (CFA) is in general modelled by large-scale Mixed-Integer Nonlinear Programs which are very challenging to be solved exactly. Moreover, the combination of both objectives means a trade-off between structural costs (capacity installation) and congestion 
costs (routing decisions) as the former tends to induce a low-cost sparse network and the latter, a less congested dense and multi-path network (see [17]).

After recalling negative-cycle optimality conditions for single and multicommodity flow networks in Section 2, we present a survey of fixed cost network design problems which are currently modelled as mixed-integer multicommodity flow problems. We consider different levels of complexity, from the pure fixed cost case to general nonconvex design cost functions, but do limit the study to flow and capacity constraints without additional topological constraints. We will consider in Section 4 a continuous but piecewise convex model for capacity expansion in a network and propose some exact local and global schemes to solve it. Decomposition among commodities is the main directive idea of many algorithms which will be compared on medium and large-scale instances of the nonconvex multicommodity flow continuous models. Besides the guarantees given by local optimality conditions on feasible cycles, these approaches take profit of the existence of performant algorithms for convex cost multicommodity network flow problems, able to produce sharp lower bounds and nice starting solutions for further local improvements.

\section{NEGATIVE CYCLE OPTIMALITY CONDITIONS}

We will analyse in the next section the optimality conditions for general cost multicommodity flow problems, focussing on the difficulty to extend the classical results for single commodity flows.

\subsection{Convex costs in single and multicommodity flows}

We consider first the so-called Negative-cycle optimality conditions, well-known for singlecommodity flow and examine to what extent they may be generalised to the multicommodity case. In their simpler form, these first-order optimality conditions state that a feasible flow is optimal if and only if there does not exist augmenting cycles with negative cost. Here, an augmenting cycle is a cycle of the graph such that any arc in the cycle possess a positive residual capacity (i.e., the total flow is strictly lower than the capacity on any forward arc and strictly positive on any backward arc of the cycle, see [1] for instance). Several authors have considered early the extension to separable convex cost functions, see [15, 52, 55, 71, 82] and [91].

To be more precise, let us recall the optimality conditions for single commodity flow problems with convex arc costs (a complete proof can be found in [15]).

Notation: for a given cycle $\Theta$ of $\mathcal{G}$ and an arbitrary sense of circulation which defines a partition of $\Theta$ in two subsets of arcs, $\Theta^{+}$for the direct arcs and $\Theta^{-}$for the reverse arcs, we will use the incidence vector of the cycle $\theta \in \mathbb{R}^{m}$ with components $\theta_{e}$ equal to $1,-1$ for the arcs in $\Theta^{+}, \Theta^{-}$ respectively and 0 for the others. For a given feasible flow $x$, we consider augmenting cycles as the ones which have a strictly positive residual capacity; i.e., a cycle $\Theta$ of $G$ is augmenting if and only if there exists a strictly positive $\bar{\alpha}$ such that $x+\alpha \theta$ is feasible for any $\alpha \in[0, \bar{\alpha}]$. Given a feasible cycle $\Theta$ we define its cost by:

$$
\lambda(x, \Theta)=\sum_{e \in \Theta^{+}} f_{e}^{\prime+}\left(x_{e}\right)-\sum_{e \in \Theta^{-}} f_{e}^{\prime}-\left(x_{e}\right)
$$


where $f_{e}^{\prime+}\left(x_{e}\right)$ (resp. $\left.f_{e}^{\prime}-\left(x_{e}\right)\right)$ is the right (resp. left) partial derivative of the arc cost function $f_{e}$ with respect to $x_{e}$.

GH theorem: Optimality conditions for the single-commodity case: A feasible solution is optimal if and only if there does not exist any augmenting cycle with negative cost.

The first interest in extending this result to MCF is the possibility to design easy-to-implement cycle-cancelling algorithms working on each commodity separately like a decomposition method. The second idea is to further study general continuous and piecewise smooth arc cost functions, giving some insight towards the nonconvex case.

It is already well-known that GH theorem cannot be extended so straightforward to the multicommodity case, even in the apparently simplest situation like linear-cost capacitated MCF. Indeed, the decomposition among the commodities in $\mathcal{K}$ is not possible. This of course does not mean that we are not able to produce optimality conditions from the primal and dual pairs of LP associated with MCF.

To illustrate the goals we aim at, we first illustrate the main difficulty on a simple example:

Let us consider the two-commodity flow network of Figure 1-a where both demands are equal to 1 and all arc capacities are equal to 1 . The arc cost coefficients are simply 0 for the vertical arcs and +1 for the horizontal arcs, so that the optimal solution uses the vertical arcs to send one unit of flow from each origin to each destination. But, one can verify easily that the feasible solution represented by the dotted paths shown on Figure 1-b does not present any augmenting cycle even if it is not optimal.

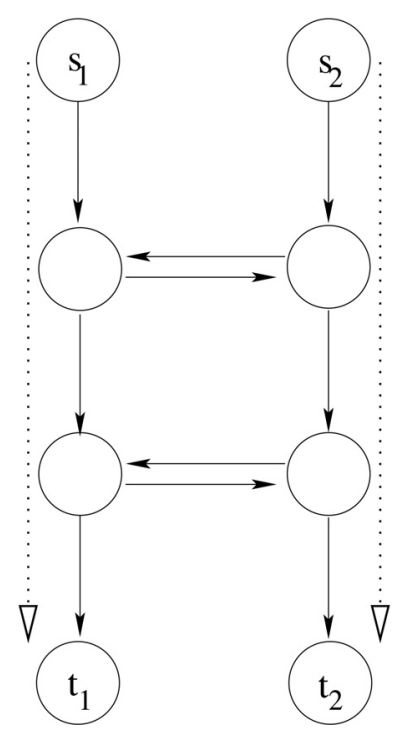

(a)

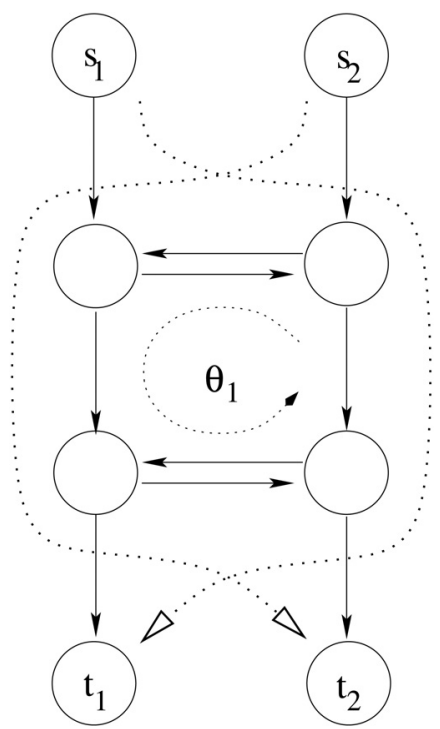

(b)

Figure 1 - The linear case does not work. 
However, it is still possible to write equivalent negative cycle conditions in the uncapacitated case with smooth convex arc cost functions. Obviously, we must add some hypotheses to ensure that an optimal solution indeed exists, like using strongly convex cost functions or coercivity assumptions. Ouorou \& Mahey [76] have shown that it is possible to extend the negative cycle optimality condition to capacitated multicommodity flow problems using arc cost functions satisfying the following property:

A: Properties of congestion functions Let consider functions $\Phi: C \times \mathbb{R} \mapsto \mathbb{R} \bigcap\{+\infty\}$ such that:

1. $\Phi(c, \cdot)$ is strictly convex, continuously derivable and monotone increasing on $(0, c)$

2. $\Phi(c, 0)=0$ and $\Phi(c, x) \rightarrow+\infty$ if $x \downarrow c$

Observe that the cost function acts as a barrier and, assuming that a strictly feasible solution exists, we can skip the capacity constraints. A well known example of such congestion function in data networks is Kleinrock's function $\Phi(c, x)=\frac{x}{c-x}$ which expresses the average delay of a traffic $x$ on an arc with capacity $c$ assuming Poissonian hypotheses for M/M/1 queues (see [17] for example).

Let $x=\sum_{k} x^{k}$ be a feasible solution of MCF such that $f(x)=\sum_{e} \Phi\left(c_{e}, x_{e}\right)$ has a finite value. We will call a cycle $\Theta k$-augmenting if it presents a strictly positive residual for commodity $k$, i.e., if we can augment the commodity flow value $x_{e}^{k}$ on the direct arcs and reduce these values on the reverse arcs. In our model, a $k$-augmenting cycle is such that all reverse arcs carry a positive value of commodity $k$.

Theorem 1. Assuming the congestion functions possess the Property (A), a feasible solution $x^{*}$ is a global minimum of MCF if and only if, for all commodities in $\mathcal{K}$, there does not exist any $k$-augmenting cycle with negative cost.

Proof. See [76].

Ouorou and Mahey observed too that the result is no more valid if smoothness is not assumed. We will analyse deeper the non smooth case in the next sections, and, in particular, we will discuss the local optimality conditions for the model MCF when the arc cost functions $f_{e}$ are piecewise convex.

\subsection{Local optimality conditions for a piecewise convex MCF}

We will analyse here the special case where the arc cost function is the minimum of convex functions such that

$$
f_{e}\left(x_{e}\right)=\min \left\{\Phi_{e l}\left(c_{e l}, x_{e}\right)+\pi_{e l}, l=0, \ldots, L\right\}
$$

where each function $\Phi_{e l}$ possess the Property (A) and $\Phi^{\prime}\left(c_{e l}, x\right) \leq \Phi^{\prime}\left(c_{e, l-1}, x\right)$ for any $0 \leq$ $x<c_{e, l-1}<c_{e l}, l=1, \ldots, L$ (we can thus assume that each $\Phi_{e l}$ is a congestion function as in Theorem 1). Because the motivating example of such functions is the Capacity Expansion 
problem, which will be described in Section 4, we assume $\pi_{e 0}=0$ and $\pi_{e, l-1}<\pi_{e l}, \forall e \in$ $\mathcal{E}, l=1, \ldots, L$. We denote in the sequel by CCE the problem of minimising the sum of arc cost functions given by (1) subject to the MCF constraints. CCE is a piecewise convex MCF.

Thanks to the simple separable structure of the cost function, it is possible to put down first-order local optimality conditions for problem CCE even in the presence of breakpoints where the cost function is not differentiable. Indeed, left and right partial derivatives do exist with respect to all variables. This implies that directional derivatives exist in all directions, allowing to use the first-order conditions for a local minimum: if $x^{*}$ is a local minimum of the function $f$, then the directional derivative $f^{\prime}\left(x^{*} ; d\right)$ is non negative in all feasible directions $d$. We will show below that the convexity of the $\Phi_{e l}$ functions that build the objective function $f$ on each arc not only allows us to characterise that condition using left and right derivatives but also turns the condition necessary and sufficient.

For simplicity of the exposition, we assume $L=1$, and thus there is one breakpoint for each arc $e$ expressed by a percentage $\gamma_{e}$ of $c_{0 e}$, i.e., $\Phi_{e}\left(c_{0 e}, x_{e}\right)=\Phi_{e}\left(c_{1 e}, x_{e}\right)+\pi_{e}$ for $x_{e}=\gamma_{e} c_{0 e}$. For any such local optimum, let define:

$$
\begin{aligned}
E_{0} & =\left\{e \in \mathcal{E} \mid x_{e}^{*} \in\left[0, \gamma_{e} c_{0 e}\right)\right\} \\
E_{1} & =\left\{e \in \mathcal{E} \mid x_{e}^{*} \in\left(\gamma_{e} c_{0 e}, c_{1 e}\right)\right\} \\
G & =\left\{e \in \mathcal{E} \mid x_{e}^{*}=\gamma_{e} c_{0 e}\right\}
\end{aligned}
$$

and let $g=|G|$. There are $2^{g}$ different partitions of the set $G$ in two disjoint subsets of arcs $G=G_{0 i} \cup G_{1 i}, i=1, \ldots, 2^{g}$, so that we can define $2^{g}$ subregions of the feasible set, denoted by $C_{i}$ :

$$
\begin{gathered}
C_{i}=\left\{x \text { feasible to MCF } \mid x_{e} \in\left[0, \gamma_{e} c_{0 e}\right] \text { for } e \in E_{0} \bigcup G_{0 i}\right. \\
\left.\qquad x_{e} \in\left[\gamma_{e} c_{0 e}, c_{1 e}\right] \text { for } e \in E_{1} \bigcup G_{1 i}\right\}
\end{gathered}
$$

These subregions have disjoint interior points and cover the feasible set of solutions of CCE in a neighbourhood of $x^{*}$. They are defined such that $x^{*} \in C_{i}, \forall i=1, \ldots, 2^{g}$. Moreover, the objective function $f$ is convex when restricted to any region $C_{i}$ and we can write optimality conditions separately in each one of these regions. Indeed, we can associate with each arc in the partition its 'active' congestion functions, i.e., $\Phi\left(c_{0 e}, x_{e}\right)$ for $e \in E_{0} \bigcup G_{0 i}$ and $\Phi\left(c_{1 e}, x_{e}\right)$ for $e \in E_{1} \cup G_{1 i}$, so that $f(x)$ is simply the sum of the active functions for $x \in C_{i}$.

\section{Kuhn-Tucker conditions on set $C_{i}$}

We define by $P_{k}$ the set of directed paths joining the corresponding origin and destination of a commodity $k \in \mathcal{K}$. Let $x_{k p}$ be the amount of flow of commodity $k$ routed on the path $p \in P_{k}$ in the local optimum $x^{*}$. There exist multipliers $u_{e}^{i}$ and $v_{k}^{i}$ satisfying:

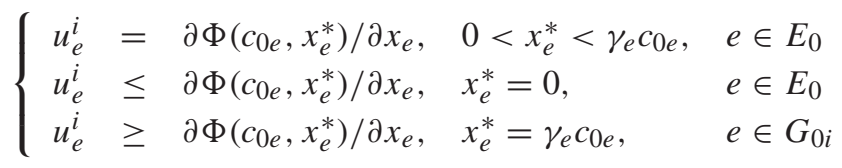




$$
\left\{\begin{array}{lll}
u_{e}^{i}=\partial \Phi\left(c_{1 e}, x_{e}^{*}\right) / \partial x_{e}, & \gamma_{e} c_{0 e}<x_{e}^{*}<c_{1 e}, & e \in E_{1} \\
u_{e}^{i} \leq \partial \Phi\left(c_{1 e}, x_{e}^{*}\right) / \partial x_{e}, & x_{e}^{*}=\gamma_{e} c_{0 e}, & e \in G_{1 i}
\end{array}\right.
$$

and, for all commodity $k$ :

$$
\begin{aligned}
& \forall p \in P_{k}, \quad x_{k p}>0, \quad v_{k}^{i}=\sum_{e \in p} u_{e}^{i} \\
& \forall p \in P_{k}, \quad x_{k p}=0, \quad v_{k}^{i} \leq \sum_{e \in p} u_{e}^{i}
\end{aligned}
$$

Recall that these conditions imply that the active paths have minimal lengths with respect to first derivatives of the active functions associated with $C_{i}$. The objective function being convex on that region, the conditions are necessary and sufficient. Thus, at a local minimum, these conditions must be satisfied for all subregions. A crucial question is then to identify situations where the solution is blocked at some breakpoint which cannot be optimal. Indeed, it can be shown that, when an arc flow is set to the breakpoint value at an optimal solution, that arc must belong to all active paths (i.e., $x_{k p}>0$ ) for all commodities using it.

Theorem 2. If $x^{*}$ is a local minimum of CCE, then every arc e such that $x_{e}^{*}=\gamma_{e} c_{0 e}$ belongs to every active path of every commodity routed through arc $e$.

Proof. Let us assume there is a commodity $k$ with two active paths $p$ and $p^{\prime}$ in $x^{*}$ such that the only arc $\hat{e}$ with $x_{\hat{e}}^{*}=\gamma_{\hat{e}} c_{0 \hat{e}}$ belongs to path $p$ and not to path $p^{\prime}$. Let us also assume that $\hat{e} \in G_{01}$ for $C_{1}$ and $\hat{e} \in G_{11}$ for $C_{2}$. On one hand, analysing active path $p^{\prime}$, we have that $v_{k}^{1}=v_{k}^{2}$. On the other hand, analysing active path $p$, we have that $v_{k}^{1}=\sum_{e \in p} u_{e}^{1}>\sum_{e \in p} u_{e}^{2}=v_{k}^{2}$, since $u_{\hat{e}}^{1} \geq \partial \Phi\left(c_{0 \hat{e}}, x_{\hat{e}}^{*}\right) / \partial x_{\hat{e}}>\partial \Phi\left(c_{1 \hat{e}}, x_{\hat{e}}^{*}\right) / \partial x_{\hat{e}} \geq u_{\hat{e}}^{2}$, and the initial hypothesis is contradicted.

Thus, a breakpoint corresponds to a kind of bottleneck arc where the total traffic is exactly equal to the breakpoint value, i.e., $x_{e}^{*}=\sum_{k \in \mathcal{K}_{e}} b_{k}=\gamma_{e} c_{0 e}$, where $\mathcal{K}_{e} \subseteq \mathcal{K}$ is the set of commodities for which arc $e$ belongs to every active path joining their corresponding origin and destination. Note that a breakpoint is not necessarily a bottleneck in the topology of the network, but rather in terms of attractiveness to route commodities since alternative paths may exist in the network but are not used. Thus, any perturbation of one of the demands flowing through arc $e$ will shift the arc flow value by the same quantity and consequently get out of the breakpoint. That observation tends to induce the fact that the number $g$ of breakpoints at a local minimum will remain quite low.

\section{Negative cycle optimality conditions}

Theorem 3. A feasible solution $x^{*}$ is a local minimum of CCE if and only if, for all commodities in $\mathcal{K}$, there does not exist any $k$-augmenting cycle with negative cost.

Proof. See [67]. 
Observe that the key fact which leads to the main result is that, at the breakpoints, $f_{e}^{\prime+}\left(x^{*}\right)<$ $f_{e}^{\prime}-\left(x^{*}\right)$ and the result could not have been extended to a convex non smooth congestion function as already observed in [76]. As an illustration, let us come back to the two-commodity flow example described in Section 2.1. We will compare two uncapacitated situations with different piecewise linear functions on the vertical arcs (the first one convex and the second one concave as shown on Figure 2) and the same linear cost $f_{e}\left(x_{e}\right)=x_{e}$ on the horizontal arcs so that the optimal solution is still to route both commodities on the vertical arcs. In a first case, the arc cost functions are given by

- $f_{e}\left(x_{e}\right)=x_{e}$ for the horizontal arcs

- $f_{e}\left(x_{e}\right)=\max \left\{1,2 x_{e}-1\right\}$ for the vertical arcs

Thus $f$ is a convex function but non smooth at $x_{e}=1$. Again, let us take the feasible but non optimal solution of Figure 1-[b]. However, there are no negative $k$-augmenting cycles for both commodities $^{1}$. We can check in particular that the cost of cycle $\Theta_{1}$ for commodity 1 is equal to 0 .

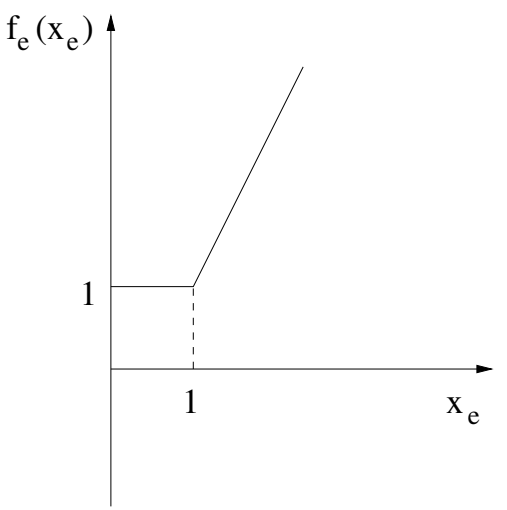

(a)

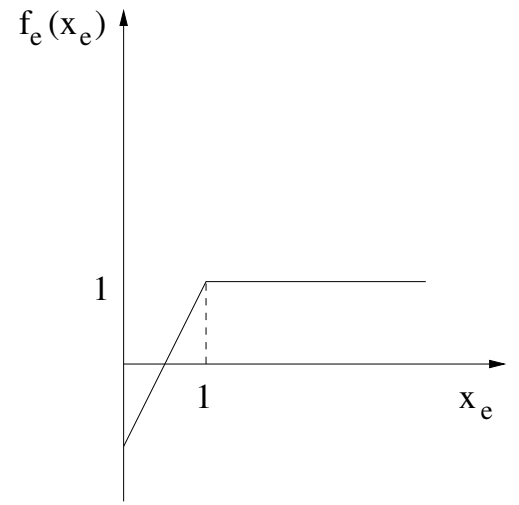

(b)

Figure 2 - Convex and concave arc costs.

In the second case, the arc cost function is

- $f_{e}\left(x_{e}\right)=x_{e}$ for the horizontal arcs

- $f_{e}\left(x_{e}\right)=\min \left\{1,2 x_{e}-1\right\}$ for the vertical arcs

Each arc-cost function is now concave piecewise linear and, considering the same solution as before, we can now find a negative cost cycle, for instance, for the first commodity, the cycle $\Theta_{1}$ has cost -4 (Fig. 2-[b]). The relation between left and right derivatives at the breakpoint is crucial to determine whether we can use the negative-cycle optimality condition or not.

${ }^{1}$ This counterexample for the convex case is due to E. Tardos [87] 


\section{FROM FIXED-CHARGE TO MULTIPLE CHOICE NETWORK DESIGN}

Our basic separable arc-cost model includes many well-studied situations like concave-cost or fixed-cost network design that we will briefly survey here before extending to more complex functions like piecewise nonlinear or step increasing discontinuous cost functions. As many interesting surveys already exist on different subjects, we will not try to be exhaustive but mainly focus on strategies which aim at decomposing among commodities. Most of the contributions, well reported in Pardalos and Rosen's survey [79], focus on Branch-and-Bound like approaches applied to single-commodity or transshipment models, extending too to location problems and Steiner trees.

General concave-cost network flow problems have been largely studied since the early results by Tuy [89] and Zangwill [93]. Minimising a concave function on a polyhedron is known to be a NP-hard problem in the general case (see [90] for some polynomial algorithms with seriesparallel networks, see too [80]) and early algorithms have relied on Branch-and-Bound associated with linearisation techniques (see $[44,92]$ ) or greedy heuristics (see $[6,70]$ ). Applications to packet-switched communications networks have been early studied by Gerla and Kleinrock [42] where they separated the design and routing costs and observed that a global minimum can be reached when the concave cost function follows a power law $f_{e}\left(x_{e}\right)=a_{e} x_{e}^{\alpha}+b_{e}$. See too [2] for mixed-integer formulations of the piecewise linear and nonlinear concave functions and use of Lagrangian Relaxation. Lagrangian heuristics have too been tested with relative success $[74,85]$. A comprehensive survey can be found in [12].

We now discuss the Fixed-Charge Uncapacitated Network Loading problem (FCUNL) which is too a basic brick in the modeling of challenging network design problems. By the way, the piecewise linear concave cost network flow problem can be modeled as a FCUNL as shown in [54], at the cost of increasing the number of arc decision variables. On the other hand, any FCUNL model can be viewed as a step or piecewise affine cost network flow problem. The cost function is generally represented by the following discontinuous function:

$$
f_{e}\left(x_{e}\right)= \begin{cases}F_{e}+h_{e} x_{e} & \text { for } x_{e}>0 \\ 0 & \text { for } x_{e}=0\end{cases}
$$

It is then generally approximated by a concave piecewise affine function for a small value $\epsilon_{e}>0$ as shown in Figure 3.

An efficient procedure based on a dual-ascent method to solve FCUNL has been proposed by Balakrishnan et al. [5]. The problem turns to be much more complex when capacities bound the flow on each arc. The main reason is that the continuous relaxation of the capacitated model is quite weak as discussed below while the uncapacitated polytope is very close to be integral (see [48]).

Fixed-charge capacitated multicommodity network flow problems have been mostly studied in the eighties and nineties decades. We send back the reader to the relatively recent survey by Gendron et al. [41] and the references therein. Modelling the problem as a mixed-integer program 


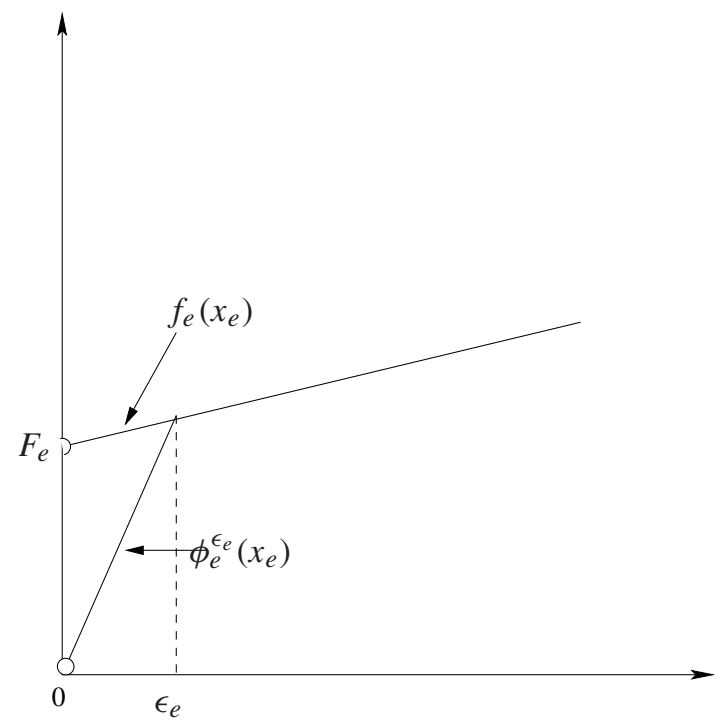

Figure 3 - Piecewise affine concave approximation.

substitutes the difficulty of handling piecewise linear approximations and concave cost functions by the introduction of integer variables. The arc cost function is thus $f(x, y)=\sum_{k} \sum_{e} h_{e k} x_{e}^{k}+$ $\sum_{e} F_{e} y_{e}$ with $y_{e} \in\{0,1\}$ and we add the following coupling inequalities:

$$
\sum_{k} x_{e}^{k} \leq c_{e} y_{e}, \forall e \in \mathcal{E}
$$

where $c_{e}$ is the capacity of $\operatorname{arc} e$.

Lagrangian Relaxation has been applied by different authors to exploit the underlying structure of the model, mainly in two directions: relaxing the coupling capacity constraints to decompose by commodity and obtain shortest-path subproblems or relaxing the flow conservation constraints for all commodities to decompose by arcs and obtain knapsack subproblems. It is well-known (see [41] for a complete analysis) that the Lagrangian lower bound is equal to the continuous relaxation bound which can be quite poor and a much better bound is obtained with reduced additional costs by forcing the so-called strong inequalities

$$
x_{e}^{k} \leq q_{e k} y_{e}, \forall e \in \mathcal{E}, \forall k \in \mathcal{K}
$$

where $q_{e k}$ is the maximum flow allowed on arc $e$ for commodity $k$ (i.e., the demand $b_{k}$ if no individual capacities are imposed on arc $e$ for commodity $k$ ).

Solving the Lagrangian dual problem can be a hard task when the number of dual multipliers increases and this has motivated the use of sophisticated subgradient algorithms like bundle methods [35] or the volume algorithm [8]. Crainic et al. [28] have reported extensive computational results with the bundle method on a large set of instances with up to 30 nodes, 700 arcs 


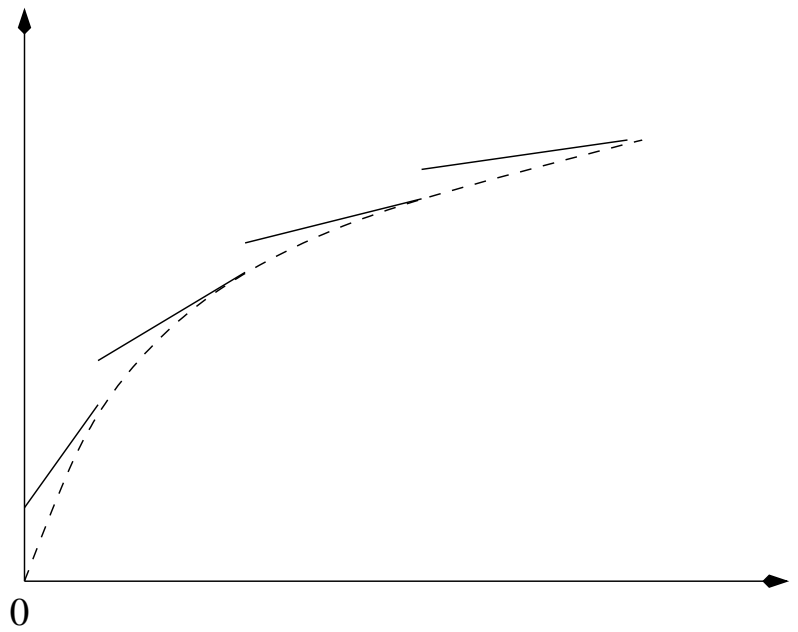

Figure 4 - Piecewise affine increasing function.

and 400 commodities. A rather surprising fact is that the 'knapsack relaxation' performs better, probably because the min-cost flow subproblems in the 'capacity relaxation' are highly degenerate. As usual, the gap can be reduced by adding valid inequalities if their separation procedure is not too costly. Further reduction of the gap to compute exact solutions of MCF needs branching and the construction of Branch-and-Cut algorithms. The polyhedral structure of the multicommodity flow solution set has been studied by various authors (see [9, 61, 86]). Bienstock and Günlük [18] have analysed linear capacitated network design problems and they gave in [19] a set of valid inequalities for the MCF-polytope, results which led to a Branch-and-Cut algorithm (see too [20]).

Heuristic approaches have been too applied to network design problems, including capacitated MCF, to obtain very reduced gaps on large instances (see [16, 27, 49, 53]). Lagrangian heuristics are able to produce nice feasible solutions on these instances by branching from the fractional nearly feasible solution given by the bundle or the volume algorithms (see $[50,56]$ ).

Telecommunications network design problems, dealing with packet-switched traffic on large multicommodity networks, have motivated the study of designing multiple facilities on the candidate arcs, turning the complexity of these models even harder. General capacitated network loading with two type of capacities has been modelled by Magnanti et al. [62].

In the general case of linear transportation costs combined with discrete prices for each facility, we obtain an equivalent piecewise affine increasing but discontinuous function (see Figure 4 for a typical profile with economies of scale). Specific valid inequalities can be devised for these cases like the residual capacity inequalities (see $[3,36])$. That general model includes the well-studied case of step increasing cost functions. Croxton et al. [29] have proved equivalence of different model structures for the piecewise linear cost case and shown their direct link with the lower 
convex envelope of the discontinuous function (i.e., the function which epigraph is the convex hull of the epigraph of the nonconvex original cost function). Different algorithmic approaches have been used in practice, see in particular [30, 40] and [58], the latter authors exploring a DC (difference of convex functions) model of the piecewise linear function (see too $[39,68]$ ).

Another direction of active research to solve capacitated network design problems has been the use of Benders decomposition to derive dual subproblems and new family of valid cuts (see [25, 41] for a general presentation and [24] for a survey on the uncapacitated and capacitated fixed-charge design problems) and various enhancements of that classical approach have been motivated by the network design models (see [26, 32, 62, 63, 83]). Generalized Benders decomposition can be too an interesting solution procedure to exactly solve difficult capacity and flow assignment problems with convex flow costs [64], as the subproblems reduce to convex multicommodity network flow problems for which efficient algorithms have been proposed (see [77] for a survey). In [45] non-convex, non-linear multicommodity flow problems issued as special cases of the capacity and flow assignment problem are solved by a Separable Augmented Lagrangian Algorithm. We will get back to these nonlinear models studying the capacity expansion problem in the next section.

Finally, we observe that MCF is a special case of general MINLP (Mixed-Integer Nonlinear Programming) for which recent developments are promising (see [46] and [22] for a survey). Many potential applications of these new algorithms have a potential multicommodity structure like water networks [21], gas networks [4, 69], energy networks [31, 75] or transportation networks [38], and naturally communications networks remain a very rich field for challenging network design problems (see for example [23] and [73]).

We will now consider specific contributions to the special situation where we want to expand (and buy) capacities on some arcs of a formerly dimensioned network to support additional demand across the network.

\section{A CONTINUOUS MODEL FOR CAPACITY EXPANSION}

A few authors have considered the case where some existing topology with already installed link capacities do exist and we want to expand these capacities to face an increasing traffic or to reduce congestion at minimal cost (see $[13,43,78])$. Of course that situation includes the general case but we focus here on the situation where the initial fixed cost is zero.

\subsection{Continuous vs. discrete models in network design}

Back to model MCF, we will use in parallel the implicit arc-path model which is designed in the following classical way.

Recall (c.f., Section 2.2) that for a given a commodity $k$, we consider the set of directed paths $P_{k}$ joining the corresponding origin and destination, and $x_{k p}$ the amount of flow of commodity $k$ through the path $p \in P_{k}$. This set may be the set of all simple directed paths or a restricted set 
of feasible paths, for instance with a limited number of hops. Let $a_{k p}$ be the arc-path incidence vector defined by

$$
a_{k p}^{e}= \begin{cases}1 & \text { if arc } e \in p \\ 0 & \text { otherwise }\end{cases}
$$

Each component $x_{e}$ of the vector $x$ denotes the total flow on $\operatorname{arc} e$. Then

$$
x_{e}=\sum_{k \in \mathcal{K}} \sum_{p \in P_{k}} a_{k p}^{e} x_{k p} .
$$

The set of multicommodity flow vectors, denoted by $\mathcal{M}(G, T)$, can be described by the implicit arc-path formulation, i.e., for each commodity $k$ flowing between nodes $s_{k}$ and $t_{k}$ the active paths must satisfy $\sum_{p \in P_{k}} x_{k p}=b^{k}$.

We assume now that each arc in the topology is expandable to a capacity $c_{1 e}>c_{0 e}$ at a given fixed cost $\pi_{e}$. Let $\delta_{e}=c_{1 e}-c_{0 e}$ be the increment of capacity. The capacity expansion model will minimise the total congestion cost plus the expansion fixed costs. Using the previously defined arc congestion cost functions $\Phi\left(c_{e}, x_{e}\right)$, we can define first a mixed-integer nonlinear model for the capacity expansion problem:

$$
\begin{aligned}
\text { Minimize } & \sum_{e}\left[\Phi\left(c_{0 e}+\delta_{e} y_{e}, x_{e}\right)+\pi_{e} y_{e}\right] \\
\text { subject to } & x \in \mathcal{M}(G, T) \\
& x_{e} \leq c_{0 e}+\delta_{e} y_{e}, \forall e \in \mathcal{E} \\
& y_{e} \in\{0,1\}, \forall e \in \mathcal{E}
\end{aligned}
$$

We will now study the relationship between DCE and a continuous model which gets rid of any boolean decision variables $y$ :

$$
\begin{aligned}
& \text { Minimize } \sum_{e} f_{e}\left(x_{e}\right)=\min \left\{\Phi\left(c_{0 e}, x_{e}\right), \Phi\left(c_{1 e}, x_{e}\right)+\pi_{e}\right\} \\
& \text { (CCE) subject to } x \in \mathcal{M}(G, T) \\
& x_{e} \leq c_{1 e}, \forall e \in \mathcal{E}
\end{aligned}
$$

\section{Remarks.}

1. As shown on Figure 5 where the nonconvex resulting arc cost function of CCE is represented, we denote by $\gamma_{e} c_{0 e}$ with $0<\gamma_{e}<1$, the breakpoint at which expansion occurs. $\gamma_{e}$ can thus be interpreted as the relative congestion of an arc beyond which the network manager is willing to pay for expansion. Thus $\pi_{e}=\Phi\left(c_{0 e}, \gamma_{e} c_{0 e}\right)-\Phi\left(c_{1 e}, \gamma_{e} c_{0 e}\right)$ is the expansion price converted in congestion cost units.

2. The arc cost function in CCE is continuous but nonconvex and non smooth at the breakpoint $\gamma_{e} c_{0 e}$. It is shown in [60] how one can easily compute a lower bound on the optimal value of CCE by convexifying each arc cost function and summing up the resulting gaps.

Trivially, if $(x, y)$ is feasible for DCE, $x$ is feasible for CCE. The following lemma is a direct consequence of the cost structure of DCE. 


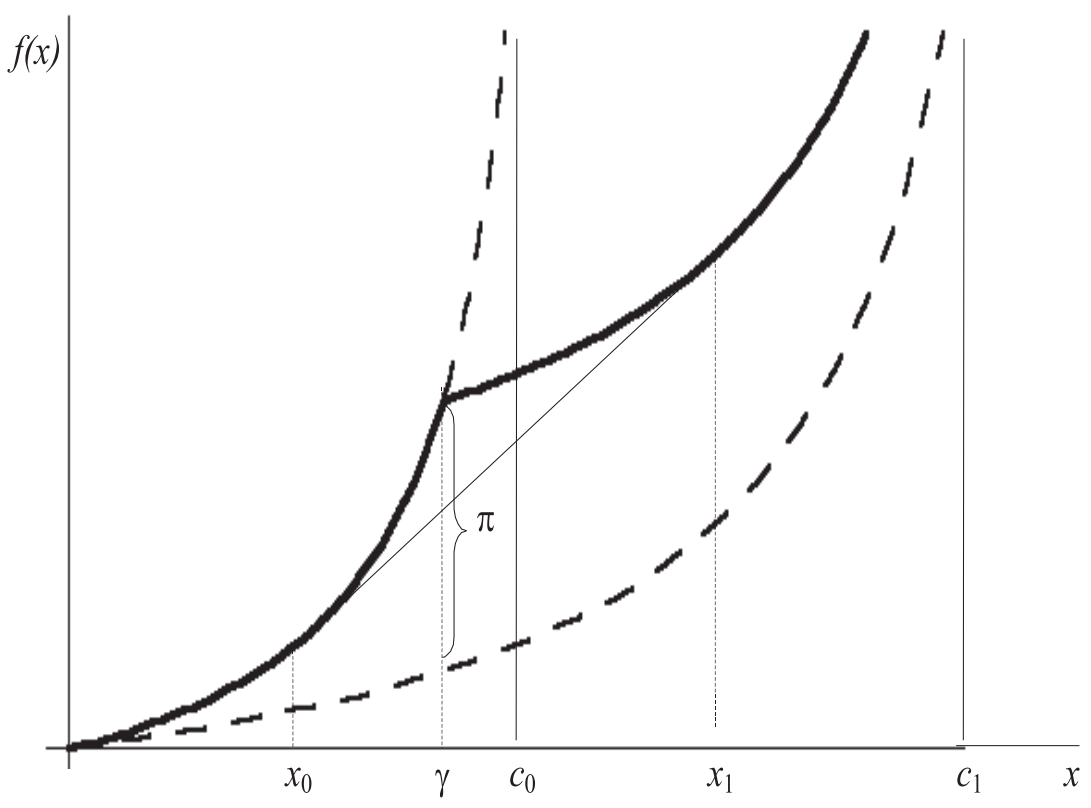

Figure 5 - The integrated function of congestion and expansion costs.

Lemma 1. Let $\left(x^{*}, y^{*}\right)$ be an optimal solution of DCE; then, we have the correspondences:

$$
\begin{aligned}
& x_{e}^{*}>\gamma_{e} c_{0 e} \Longrightarrow y_{e}^{*}=1 \\
& x_{e}^{*}<\gamma_{e} c_{0 e} \Longrightarrow y_{e}^{*}=0
\end{aligned}
$$

Moreover, if there exists an arc e with $x_{e}^{*}=\gamma_{e} c_{0 e}$, then $y_{e}^{*}$ can be either 0 or 1 , so the optimal solution is not unique.

Proof. The two cases where $x_{e}^{*}$ is not a breakpoint are straightforward. If $x_{e}^{*}=\gamma_{e} c_{0 e}$, we have:

$$
\Phi\left(c_{0 e}, \gamma_{e} c_{0 e}\right)=\Phi\left(c_{1 e}, \gamma_{e} c_{0 e}\right)+\pi_{e}
$$

which shows that the value of the arc cost function does not change whenever $y_{e}^{*}$ is 0 or 1 .

The correspondence between optimal solutions of DCE and CCE follows immediately:

\section{Theorem 4.}

i) If $\left(x^{*}, y^{*}\right)$ is an optimal solution of DCE, then $x^{*}$ is optimal for CCE and the cost values are equal. 
ii) If $x^{*}$ is an optimal solution of CCE, then $\left(x^{*}, y^{*}\right)$ is optimal for DCE with:

$$
y_{e}^{*}\left\{\begin{array}{lll}
=0 & \text { if } & 0 \leq x_{e}^{*}<\gamma_{e} c_{0 e} \\
=1 & \text { if } & \gamma_{e} c_{0 e}<x_{e}^{*}<c_{1 e} \\
\in\{0,1\} & \text { if } & x_{e}^{*}=\gamma_{e} c_{0 e}
\end{array}\right.
$$

Observe that these results apply to optimal solutions. Feasible solutions for DCE where $x_{e}<$ $\gamma_{e} c_{0 e}$ and $y_{e}=1$ or $\gamma_{e} c_{0 e}<x_{e}<c_{0 e}$ and $y_{e}=0$ have no counterpart on CCE, but the above results show that these solutions can never be optimal. We have analysed before local optimal solutions of CCE (c.f., Section 2.2). The concept of a local optimal solution of DCE is not clearly defined because of the discrete nature of variables $y$. But using the correspondence defined above in Theorem 4 part ii), we can define such a local optimum for DCE.

Finally, we would like to point out that the tight relationship between the optimal solutions of both models does not mean that they are equivalent. The continuous model is in general not able to take in consideration additional constraints on the topology which, in the contrary, can be generally done by the $y$-variables. Nevertheless, we will mention a few common situations where it is possible to convert such constraints from DCE to CCE:

a. Many models of network design require symmetry of the link capacities. This is easily modelled in DCE by the constraint $y_{i j}=y_{j i}$ for some $\operatorname{arc} e=(i, j)$. To obtain the same effect, we must add the following constraint in CCE:

$$
\left(x_{i j}-\gamma_{i j} c_{0 i j}\right)\left(x_{j i}-\gamma_{j i} c_{0 j i}\right) \geq 0
$$

b. Cutset constraints: Let $A$ be a subset of nodes of $V$ and $C_{A}$ the corresponding cutset. Forcing the subset $A$ to be connected to the other nodes by at least one expanded arc can be modelled in DCE by $\sum_{e \in C_{A}} y_{e} \geq 1$, which is equivalent in CCE to:

$$
\max _{e \in C_{A}} \frac{x_{e}}{\gamma_{e} c_{0 e}} \geq 1
$$

Observe that both constraints derived in a. and b. define polyhedral nonconvex regions of $\mathbb{R}^{|\mathcal{E}|}$.

\subsection{Local minimisation by cycle-cancelling algorithm}

Based on the local optimality conditions described above, a cycle-cancelling algorithm has been derived in [66] (see also [84]) with two main characteristics:

- Successive cycle cancelling steps are performed by moving the flow of one commodity at a time, so that the algorithm is a decomposition method.

- Nonlinear and non smooth arc cost functions are allowed, as long as right derivatives are not greater than left derivatives at the breakpoints. 
The algorithm makes use of the concept of $k$-feasible negative cycles where it is allowed to increase strictly the $k$-th flow and thus strictly decrease the cost function. Referred to as CCA in the following, it includes an adaptation of Barahona-Tardos [10] technique to select the most negative family of node-disjoint cycles.

The algorithm is resumed below:

\section{Algorithm CCA}

- Find a feasible initial solution $x^{0}$. Set $t=0$.

- If there exists no $k$-feasible cycle with negative cost, then stop: $x^{t}$ is a local minimum for CCE.

- For some $k$, let $\Theta_{t}$ be a $k$-feasible cycle such that $\lambda\left(x^{t}, \Theta_{t}\right)<0$ and, for each arc $e \in \Theta_{t}$, compute the greatest step $\alpha_{e}$ such that:

$$
\left\{\begin{array}{l}
f_{e}^{\prime}-\left(x_{e}^{t}+\alpha_{e}\right) \leq f_{e}^{\prime}\left(x_{e}^{t}\right)-\lambda\left(x^{t}, \Theta_{t}\right) \\
\quad \text { if } e \in \Theta_{t}^{+} \text {and } x_{e}^{t} \geq \gamma c_{0 e} \\
f_{e}^{\prime}-\left(x_{e}^{t}+\alpha_{e}\right) \leq f_{e}^{\prime}\left(x_{e}^{t}\right)-\lambda\left(x^{t}, \Theta_{t}\right) \text { and } \alpha_{e} \leq \gamma c_{0 e}-x_{e}^{t} \\
\text { if } e \in \Theta_{t}^{+} \text {and } x_{e}^{t}<\gamma c_{0 e} \\
f_{e}^{\prime}+\left(x_{e}^{t}-\alpha_{e}\right) \geq f_{e}^{\prime}-\left(x_{e}^{t}\right)+\lambda\left(x^{t}, \Theta_{t}\right) \text { and } \alpha_{e} \leq \min \left\{x_{e}^{k t}, x_{e}^{t}-\gamma c_{0 e}\right\} \\
\text { if } e \in \Theta_{t}^{-} \text {and } x_{e}^{t}>\gamma c_{0 e} \\
f_{e}^{\prime+}\left(x_{e}^{t}-\alpha_{e}\right) \geq f_{e}^{\prime}-\left(x_{e}^{t}\right)+\lambda\left(x^{t}, \Theta_{t}\right) \text { and } \alpha_{e} \leq x_{e}^{k t} \\
\text { if } e \in \Theta_{t}^{-} \text {and } x_{e}^{t} \leq \gamma c_{0 e}
\end{array}\right.
$$

- Let $\alpha^{t}=\min _{e \in \Theta}\left\{\alpha_{e}\right\}$ and set:

$$
x^{k, t+1}=x^{k t}+\alpha^{t} \theta_{t}
$$

- Set $t=t+1$ and iterate.

where $\theta_{t}$ in the update formula (3) denotes the incidence vector of the cycle $\Theta_{t}$.

The complexity of that computation is only apparent, as we can observe that, in many cases, a larger step can be performed when one reaches the breakpoint value. Indeed, suppose that $x_{e}^{t}<$ $\gamma c_{0 e}$ and that the flow augments until $x_{e}^{t}+\alpha_{e}=\gamma c_{0 e}$ with $f_{e}^{\prime}-\left(x_{e}^{t}+\alpha_{e}\right)<f_{e}^{\prime}+\left(x_{e}^{t}\right)-c\left(x^{t}, \Theta_{t}\right)$. Then, as $f_{e}^{\prime}-\left(x_{e}^{t}+\alpha_{e}\right)>f_{e}^{\prime}+\left(x_{e}^{t}+\alpha_{e}\right)$, we can still augment the flow in the interval $\left[\gamma c_{0 e}, c_{1 e}\right)$ corresponding to the adjacent subregion. That remark justifies the fact that the one-dimensional search on the negative cycle can be directly performed on the whole interval $\left[0, c_{1 e}\right)$, even if the function is nonconvex and non smooth. The situation where one arc is set to its kink value is however possible. Convergence to a local minimum is guaranteed by the following results:

Lemma 2. After each cycle cancelling step of algorithm CCA the objective function strictly decreases. 
Proof. All cycles being disjoint implies that the multidimensional function $\phi\left(\alpha_{1}, \ldots, \alpha_{L}\right)=$ $f\left(x+\sum_{l=1}^{L} \alpha_{l} \theta_{l}\right)$ is separable with respect to cycles and can be minimised separately for each disjoint cycle. The partial derivative of $\phi$ with respect to $\alpha_{l}$ at $\alpha_{l}=0$ is simply $\partial \phi / \partial \alpha_{l}(0)=$ $\lambda\left(x, \Theta_{l}\right)$, the negative cost of cycle $\Theta_{l}$.

At any cancelling step, the directional derivative is strictly negative. As the cycle is $k$-feasible, the residual capacity of the cycle is positive. But the function $\phi_{\theta}$ is strictly convex which implies that the objective function $f$ strictly decreases at each step.

The complete convergence study of the algorithm relies on the properties of descent algorithms (see $[66,84]$ for details), so that we end with the following convergence result:

Theorem 5. Suppose there exists a strictly feasible multicommodity solution to the problem with capacities $c_{1 e}$ for all $e \in \mathcal{E}$, then the sequence generated by algorithm CCA with feasible step sizes converges to a point which satisfies the local optimality conditions of CCE.

Proof. The objective function is currently continuously differentiable on the whole intervals. Then, as the direction is sufficiently decreasing by Lemma 2 and the Armijo's condition is always satisfied when the step is not limited to the interval bounds, it is a well-known result (see for instance [34]) that the method will converge and each limit point is such that the gradient of $f$ is zero or, equivalently, there are no negative cost $k$ - feasible cycles for all commodities.

Observe that, in the original paper by Weintraub [91], many assignment subproblems are solved at each step to approximate the most helpful cycle, in the sense of minimising the decrease of the objective function after the flow update. This choice was exploited later by Barahona \& Tardos [10] to obtain a polynomial algorithm in the linear case. Our choice is different as it relies on the idea of an approximation of the steepest-descent direction.

At this point it is interesting to note that the classical Capacity Assignment - Flow Assignment (CA-FA) approach for the capacity expansion problem may return a solution that is not a local optimum of CCE. The CA-FA algorithm (see [37, 42]) alternates between a capacity assignment phase with fixed routing and a flow assignment phase with fixed arc capacities until no further improvements are possible. In order to apply the CA-FA algorithm to the capacity expansion problem, we must decide which one of the two capacities $c_{0 e}$ and $c_{1 e}$ (consequently which one of the two 'active' congestion functions $\Phi\left(c_{0 e}, x_{e}\right)$ and $\left.\Phi\left(c_{1 e}, x_{e}\right)\right)$ to assign whenever an arc $e$ is at the breakpoint, i.e., $x_{e}=\gamma c_{0 e}$. Suppose, without loss of generality, that CA-FA assigns $c_{0 e}$ to such an arc. Let us assume that the routing does not change in the flow assignment phase and the algorithm stops at a solution $\bar{x}$ with an $\operatorname{arc} e$ at its breakpoint that is not a bottleneck arc. Since the capacities are fixed for each arc, the problem in the flow assignment phase is a convex MCF, and $\bar{x}$ satisfies the optimality conditions in Theorem 1 (c.f., Section 2.1). This means that $\sum_{e \in \Theta^{+}} \bar{\Phi}_{e}^{\prime}\left(\bar{x}_{e}\right)-\sum_{e \in \Theta^{-}} \bar{\Phi}_{e}^{\prime}\left(\bar{x}_{e}\right)=0$ in either sense of circulation for cycle $\Theta$, where $\bar{\Phi}_{e}$ is the 
active congestion function of arc $e$ given by the previous capacity assignment phase. But in the sense of circulation in which the arc at its breakpoint is a forward arc we have that

$$
\sum_{e \in \Theta^{+}} f^{\prime}\left(\bar{x}_{e}\right)<\sum_{e \in \Theta^{+}} \bar{\Phi}_{e}^{\prime}\left(\bar{x}_{e}\right) \text { as } f^{\prime}\left(\gamma c_{0 e}\right)<\bar{\Phi}_{e}^{\prime}\left(\gamma c_{0 e}\right),
$$

and the solution $\bar{x}$ returned by CA-FA is not a local optimum of CCE, which would return a better solution. This happens because assigning a capacity for each arc the CA-FA restricts the search to only one of the $2^{|G|}$ regions induced by the set $G=\left\{e \in \mathcal{E} \mid \bar{x}_{e}=\gamma_{e} c_{0 e}\right\}$ (c.f., Section 2.2). On the other hand, in the CCA the $2^{|G|}$ regions are taken into account by the right and left derivates in the negative cycle optimality conditions.

We present some numerical results obtained with CA-FA and CCA. Our main interest in presenting these results is twofold: (i) to show that we can significantly improve feasible solutions obtained by convex approximation applying a local optimisation procedure; and (ii) to show that the solution obtained with CA-FA may actually not be an local optimum, which case CCA can assure local optimality.

The convex approximation proposed by Luna \& Mahey [60] is used to generate lower bounds of the global minima and initial solutions. The procedure explores the separability of the objective function by convexifying each arc cost function. It allows the use of efficient algorithms for convex multicommodity flow problems. In particular, the Proximal Decomposition method described in Mahey et al. [65] can be used to solve the convex multicommodity flow problems found in the initial convex approximation and in the routing phases of the CA-FA algorithm. Large networks with different topologies were used by Resende \& Ribeiro [81] in the context of private virtual circuit routing. In these problems, a frame relay service offers virtual private networks to customers by provisioning a set of permanent (long-term) private virtual circuits between endpoints on a large backbone network. Table 1 summarises the characteristics of the networks considered.

Table 1 - Network characteristics.

\begin{tabular}{llrrr}
\hline Instance & Topology & $|V|$ & $|E|$ & $K$ \\
\hline att & AT\&T Worldnet backbone & 90 & 274 & 272 \\
fr250 & Frame-relay & 60 & 688 & 250 \\
fr500 & Frame-relay & 60 & 906 & 500 \\
hier50 & 2-level hierarchical & 50 & 148 & 245 \\
\hline
\end{tabular}

We solved to local optimality the CCE model on the topologies shown in Table 1 fixing the ratio $c_{1 e} / c_{0 e}=4$ and the parameter $\gamma=50 \%$ (as these had been the most difficult scenarios in our preliminary experiments). Table 2 displays the results obtained when first performing CAFA and then CCA. The first three columns presents the instance identification, the demand of each commodity, and the initial capacities. We report, for the initialisation phase, the relative deviation between the cost of the optimal solution of the convexified problem in the objective function of CCE and the lower bound given by the convex approximation, and the iterations 
to solve the convex approximation with the Proximal Decomposition algorithm proposed by Mahey et al. [65]. Then, we report, the relative deviation with respect to the lower bound given by the convex approximation and the number of arcs indicated for expansion at the local optima obtained. In the two last columns, we report the total number of iterations needed by the Proximal Decomposition algorithm and, in parenthesis, the number of convex routing problems solved by the CA-FA, as well as the iterations needed by the CCA to assure local optimality ( 0 means that CA-FA obtained a local optimum).

Table 2 - Results for larger networks with $c_{1 e} / c_{0 e}=4$ and $\gamma=50 \%$.

\begin{tabular}{crrrrrrrr}
\hline & \multicolumn{4}{c}{ Initial Solution } & \multicolumn{4}{c}{ Local Optimum } \\
& $b_{k}$ & $c_{0 e}$ & $\mathrm{~d}(\%)$ & it & $\mathrm{d}(\%)$ & exp & it (CA-FA) & it (CCA) \\
\hline att & 1 & 10 & 27.5 & 2302 & 21.0 & 122 & $426(2)$ & 0 \\
fr250 & 1 & 4 & 69.0 & 191 & 42.3 & 55 & $718(4)$ & 0 \\
fr250 & 2 & 4 & 61.8 & 177 & 33.7 & 189 & $1933(5)$ & 120 \\
fr500 & 1 & 4 & 65.8 & 255 & 39.8 & 162 & $902(3)$ & 0 \\
fr500 & 2 & 4 & 41.8 & 234 & 27.2 & 387 & $6796(11)$ & 0 \\
hier50 & 1 & 10 & 36.6 & 1270 & 29.4 & 86 & $3715(4)$ & 53 \\
hier50 & 2 & 10 & 9.3 & 2827 & 7.2 & 119 & $3175(3)$ & 0 \\
\hline
\end{tabular}

For these larger networks, the average and the maximum deviation reductions are $16 \%$ and $28.1 \%$ respectively. It is worth to note that in 2 out of 7 cases the solution obtained by CA-FA was not a local minimum since CCA was executed for some iterations.

\subsection{Towards global optimisation of CCE}

Encouraged by the quality of local optimal solutions, further enhancements have been proposed in [84] and [33] towards global optimisation of the capacity expansion model.

In the first reference [84], the CCA was embedded in a tabu search algorithm to explore the solution space beyond the first local optimum. The authors reported significant improvements in a majority of instances, mainly when the initial local optimum presented more arcs at the breakpoints values. Basically, the tabu search works in two distinct phases. In the first phase, the algorithm accepts a cost increasing solution by driving the search from a local optimum $x^{*}$ to the boundary of one of the subregions composing the neighbourhood of $x^{*}$. This is achieved by pushing flow around positive-cost cycles to bring the flow on an arc $e$ to its breakpoint $\gamma_{e} c_{0 e}$. In the second phase, CCA is applied. The use of a short-term memory structure ensures that CCA does not get back to $x^{*}$, and leads the descent to a different local optimum.

In [33], the authors proposed an implicit enumeration scheme which was tested on a large set of nonconvex instances of CCE. These tests include comparisons with global solvers like BARON [88] and LINDO Global [59]. The implicit enumeration algorithm combines information from both DCE and CCE. A partial solution defines the capacities of a subset of arcs assigning values to the corresponding binary variables of DCE. A key feature of implicit enumeration 
is the ability to generate information that can be used to exclude all the completions of a partial solution from further consideration. This is done with CCE, which is used either to provide lower and upper bounds on the value of the best feasible completion or to show that there is no feasible completion.

Gürel [47] addressed CCE by branch-and-bound where the congestion cost function is a convex increasing power function. The problem is then reformulated as a conic mixed integer programming problem and solved by using branch-and-bound softwares equipped with efficient secondorder conic programming algorithms. To reformulate the problem as a second-order conic programming problem the convex terms in the objective function are first moved to constraints by adding a set of auxiliary variables bounding their values. The resulting problem has a linear objective function but a set of non-linear constraints, which are then replaced by conic quadratic inequalities. Numerical results reported indicate that some practical size problems could be solved to the optimum.

\section{CONCLUDING REMARKS}

We have proposed a survey on nonconvex multicommodity flow problems focussing on separable continuous models which are currently solved or approximated by MINLP schemes. The main fact is that local optimality conditions can decompose by commodities in some specific situations like the piecewise convex case which is a current model in telecommunications network design with QoS driven cost functions. The copious literature on fixed-cost MCF shows that mixed-integer formulations are still the most natural modelling to treat these basic Network Design problems and their numerous variants. Nevertheless, we have shown that piecewise affine or even piecewise convex arc cost functions appear in frequent situations like the capacity expansion problem with nonlinear congestion costs and they take profit of the possibility to exploit directly the optimality conditions of the continuous non convex models. The latter lead naturally to decomposition among commodities where one can benefit from the existence of efficient computational tools for convex MCF.

\section{ACKNOWLEDGMENTS}

The authors are greatly indebted to Prof. H.P.L. Luna who was an early contributor of their research. The authors acknowledge the financial support of CNPq, the first author grant PVE 400769/2014-0 and the second author grant PQ 307360/2013-0.

\section{REFERENCES}

[1] Ahuja RV, Magnanti TL \& ORLin JB. 1993. Network Flows: Theory, Algorithms and Applications. Prentice-Hall, Englewood Cliffs, NJ.

[2] Amiri A \& Pirkul H. 1977. New formulation and relaxation to solve a concave-cost network problem. Journal of the Operational Research Society, 48: 278-287. 
[3] AтAмtÜRK A \& RAJAn D. 2002. On splittable and unsplittable flow capacitated network design arc-set polyhedra. Mathematical Programming, 92: 315-333.

[4] Babonneau F, Nesterov Y \& Vial JP. 2012. Design and operations of gas transmission networks. Operations Research, 60: 34-47.

[5] Balakrishnan A, Magnanti TL \& Wong. 1989. A dual-ascent procedure for large-scale uncapacitated network design. Operations Research, 37: 716-740.

[6] Balakrishnan A \& Graves CS. 1989. A composite algorithm for a concave-cost network flow problem. Networks, 19: 175-202.

[7] Balakrishnan A, Magnanti Tl \& Mirchandani P. 1997. Network Design, In: Dell Amico M, MafFioli F \& MARTEllo S. (eds.), Annotated Bibliographies in Combinatorial Optimization, Wiley, pp. 311-334.

[8] BARAhONA F \& ANBIL R. 2000. The volume algorithm: producing primal solutions with subgradient method. Mathematical Programming, A 87: 385-399.

[9] Barahona F. 1996. Network design using cut inequalities. SIAM Journal on Optimization, 6: 823-837.

[10] BARAHONA F \& TARDos E. 1989. Note on Weintraub's minimum-cost circulation algorithm. SIAM Journal on Computing, 18: 579-583.

[11] Barnhart C, Hane CA \& Vence PH. 2000. Using branch-and-price-and-cut to solve origindestination integer multicommodity flow problems. Operations Research, 48: 318-326.

[12] BAZLAmAÇCI CF \& SAY F. 2007. Minimum concave cost multicommodity network design. Telecommunication Systems, 36: 181-203.

[13] Bektaş T, Chouman M \& CRAinic TG. 2010. Lagrangean-based decomposition algorithms for multicommodity network design problems with penalized constraints. Networks, 55: 171-180.

[14] Bell GJ \& LAMAR BW. 1997. Solution methods for nonconvex network flow problems. In: PARDALos PM, HeARn DW \& HAGer WW. (eds.), Network Optimization. Lecture Notes in Economics and Mathematical Systems, 450: 32-50, Springer.

[15] Berge C \& Ghouila-Houri A. 1962. Programmation, Jeux et Réseaux de Transport, Dunod.

[16] Berger D, Gendron B, Potvin Jy, Raghavan S \& Soriano P. 2000. Tabu search for a network loading problem with multiple facilities. Journal of Heuristics, 6: 253-267.

[17] Bertsekas DP \& Gallager RG. 1987. Data Networks, 2nd Edition, Prentice-Hall.

[18] BIENSTOCK D \& GÜNLÜK O. 1995. Computational experience with a difficult mixed-integer multicommodity flow problem. Mathematical Programming, 68: 213-237.

[19] Bienstock D \& GÜNLÜK O. 1996. Capacitated network design - Polyhedral structure and computation. INFORMS Journal on Computing, 8: 243-259.

[20] Bienstock D, Chopra S, GÜnlük O \& Tsai C. 1998. Minimum cost capacity installation for multicommodity network flows. Mathematical Programming, 81: 177-199.

[21] Bragalli C, D’ Ambrosio C, Lee J, Lodi A \& Toth P. 2006. An MinLP solution method for a water network problem. In: AZAR Y \& ERLEBACH T. (eds.) Algorithms - ESA 2006. Lecture Notes in Computer Science, 4168: 696-707, Springer. 
[22] BURER S \& LETCHFORD AN. 2012. Non-convex mixed-integer nonlinear programming: A survey. Surveys in Operations Research and Management Science, 17: 97-106.

[23] Chiang M. 2009. Nonconvex optimization for communication networks. In: GaO D \& Sherali H. (eds.), Advances in Applied Mathematics and Global Optimization. Advances in Mechanics and Mathematics, 17: 137-196, Springer.

[24] Costa AM. 2005. A survey on Benders decomposition applied to fixed-charge network design problems. Computers and Operations Research, 32: 1429-1450.

[25] Costa AM, Cordeau JF \& Gendron B. 2009. Benders, metric and cutset inequalities for multicommodity capacitated network design. Computational Optimization and Applications, 42: 371-392.

[26] Costa AM, Cordeau JF, Gendron B \& Laporte B. 2012. Accelerating Benders decomposition with heuristic masterproblem solutions. Pesquisa Operacional, 32: 3-20.

[27] Crainic TG, Gendreau M \& Farvolden J. 2000. A simplex-based Tabu search for the multicommodity capacitated fixed charge network design. INFORMS Journal on Computing, 12: 223-236.

[28] Crainic TG, Frangioni A \& Gendron B. 2001. Bundle-based relaxation method for multicommodity capacitated fixed charge network design. Discrete Applied Mathematics, 112: 73-99.

[29] Croxton KL, Gendron B \& Magnanti TL. 2003. A comparison of mixed-integer programming models for nonconvex piecewise linear cost minimization problems. Management Science, 49 : $1268-1273$.

[30] Croxton KL, Gendron B \& Magnanti TL. 2007. Variable disaggregation in network flow problems with piecewise linear costs. Operations Research, 55: 146-157.

[31] D’ Ambrosio C, LeE J \& WÄChter A. 2012. An algorithmic framework for MINLP with separable non-convexity. In: LeE J \& LeYFFER S. (eds.), Mixed Integer Nonlinear Programming. The IMA Volumes in Mathematics and its Applications, 154: 315-347, Springer.

[32] FAKhri A \& Ghatee M. 2016. Application of Benders decomposition method in solution of a fixed-charge multicommodity network design problem avoiding congestion. Applied Mathematical Modelling, 40: 6488-6476.

[33] Ferreira RPM, Luna HPL, Mahey P \& DE Souza MC. 2013. Global optimization of capacity expansion and flow assignment in multicommodity networks. Pesquisa Operacional, 33: 217-234.

[34] Fletcher R. 1987. Practical Methods of Optimization, 2nd Edition, Wiley.

[35] Frangioni A \& Gallo G. 1999. A bundle-type dual ascent approach to linear multicommodity min cost flow problems. INFORMS Journal on Computing, 11: 370-393.

[36] Frangioni A \& Gendron B. 2009. 0-1 reformulations of the multicommodity capacitated network design problem. Discrete Applied Mathematics, 157: 1229-1241.

[37] Fratta L, Gerla M \& Kleinrock L. 1973. The flow deviation method: An approach to storeand-forward communication network design. Networks, 3: 97-133.

[38] Fügenschuh A, Herty M, Klar A \& Martin A. 2006. Combinatorial and continuous models for the optimization of traffic flow networks. SIAM Journal on Optimization, 16: 1155-1176.

[39] Gabrel V \& Minoux M. 1997. LP relaxations better than convexification for multicommodity network optimization problems with step increasing cost functions. Acta Mathematica Vietnamica, 22: $123-145$. 
[40] Gabrel V, Knippel A \& Minoux M. 1999. Exact solution of multicommodity network optimization problems with general step cost functions. Operations Research Letters, 25: 15-23.

[41] Gendron B, Crainic TG \& Frangioni A. 1999. Multicommodity capacitated network design. In: B. Sansò and P. Soriano (eds), Telecommunications Network Planning, pp. 1-19, Kluwer.

[42] Gerla M \& Kleinrock L. 1977. On the topological design of distributed computer networks, IEEE Transactions on Communications, 25: 48-60.

[43] Gerla M, Monteiro JAS \& Pazos R. 1989. Topology design and bandwith allocation in ATM nets. IEEE Journal on Selected Areas in Communications, 7: 1253-1262.

[44] Gersht A \& Weihmayer R. 1990. Joint optimization of data network design and facility selection. IEEE Journal on Selected Areas in Communications, 8: 1667-1681.

[45] Gueye O, Woungang I, Khandaker F, Alam ABMB \& Dhurandher SK. 2014. An adaptive lagrangian algorithm for solving the capacity and flow assignment problem in self-healing ATM networks. IEEE 27th Biennial Symposium on Communications, pp. 134-138.

[46] GÜNLÜK O \& LindeROTH J. 2010. Perspective reformulations of mixed-integer nonlinear programs with indicator variables. Mathematical Programming, B 124: 183-205.

[47] GÜREL S. 2011. A conic quadratic formulation for a class of convex congestion functions in network flow problems. European Journal of Operational Research, 211: 252-262.

[48] Hellstrand J, Larsson T \& Migdalas A. 1992. A characterization of the uncapacitated network design polytope. Operations Research Letters, 12: 159-163.

[49] Hewitt M, Nemhauser GL \& Savelsbergh MWP. 2010. Combining exact and heuristic approaches for the capacitated fixed-charge network flow problem. INFORMS Journal on Computing, 22: 314-325.

[50] Holmberg K \& Yuan D. 2000. A Lagrangean heuristic based branch-and-bound approach for the capacitated network design problem. Operations Research, 48: 461-481.

[51] Horst R, Pardalos PM \& Thoai NV. 1996. Introduction to Global Optimization, Kluwer Academic ed., Dordrecht.

[52] Karzanov A \& MC CoRmick ST. 1997. Polynomial methods for separable convex optimization in unimodular spaces with applications. SIAM Journal on Computing, 26: 1245-1275.

[53] Katayama N, Chen M \& Kubo M. 2009. A capacity scaling heuristic for the multicommodity capacitated network design problem. Journal of Computational and Applied Mathematics, 232: 90-101.

[54] Kim D \& Pardalos PM. 2000. Dynamic slope scaling and trust interval techniques for solving concave piecewise linear network flow problems. Networks, 35: 216-222.

[55] KLEIN M. 1967. A primal method for minimal cost flows with applications to the assignment and transportation problems. Management Science, 14: 205-220.

[56] Kliever G \& Timajev L. 2005. Relax-and-cut for capacitated network design, In: Brodal GS \& LeONARDi S. (eds.), Algorithms - ESA 2005. Lecture Notes in Computer Science, 3669: 47-58, Springer. 
[57] LAMAR BW \& WALlace CA. 1997. NETSPEAK: An algebraic modelling language for nonconvex network optimization problems. In: PARdalos PM, HeARn DW \& HAGER WW. (eds.). Network Optimization. Lecture Notes in Economics and Mathematical Systems, 450: 328-345, Springer.

[58] Lethi HA \& PhAM DT. 2002. DC programming approach for multicommodity network optimization problems with step-increasing cost functions. Journal of Global Optimization, 22: 205-232.

[59] Lin Y \& Schrage L. 2009. The global solver in the LINDO API. Optimization Methods and Software, 24: 657-668.

[60] Luna HPL \& MAHEY P. 2000. Bounds for global optimization of capacity expansion and flow assignment problems. Operations Research Letters, 26: 211-216.

[61] Magnanti TL, Mirchandani P \& Vachani R. 1993. The convex hull of two-core capacitated network design problems. Mathematical Programming, 60: 233-250.

[62] Magnanti TL, Mirchandani P \& Vachani R. 1995. Modeling and solving the two-facility capacitated network loading. Operations Research, 43: 142-157.

[63] Magnanti Tl, Mireault P \& Wong RT. 1986. Tailoring Benders decomposition for uncapacitated network design. In: GALlo G \& SANDI C. (eds.). Netflow at Pisa. Mathematical Programming Studies, 26: 112-154, Springer.

[64] Mahey P, Benchakroun A \& Boyer F. 2001. Capacity and flow assignment of data networks by generalized Benders decomposition. Journal of Global Optimization, 20: 173-193.

[65] Mahey P, Ouorou A, LeBlanc L \& Chifflet J. 1998. A new proximal decomposition algorithm for routing in telecommunication networks. Networks, 31: 227-238.

[66] Mahey P \& DE Souza MC. 2004. Solving multicommodity flow problems with separable piecewise convex costs, Research Report RR04-09, LIMOS, CNRS, Univ. Clermont Auvergne.

[67] MAHeY P \& DE SouZA MC. 2007. Local optimality conditions for multicommodity flow problems with separable piecewise convex costs. Operations Research Letters, 35: 221-226.

[68] Mahey P, Phong TQ \& Luna HLP. 2001. Separable convexification and DCA techniques for capacity and flow assignment problems. RAIRO Operations Research, 35: 269-281.

[69] Martin A, Möller M \& Moritz S. 2006. Mixed-integer models for the stationary case of gas network optimization. Mathematical Programming, 105: 563-582.

[70] Minoux M. 1976. Multiflots de coût minimal avec fonctions de coût concaves. Annales des Télécommunications, 31: 77-92.

[71] Minoux M. 1986. Solving integer minimum cost flows with separable convex cost objective polynomially. In: Gallo G \& SANDi C. (eds.). Netflow at Pisa. Mathematical Programming Studies, 26: 237-239, Springer.

[72] Minoux M. 1989. Network synthesis and optimum network design problems: Models, solution methods and applications. Networks, 19: 313-360.

[73] Morabito R, De Souza MC \& Vazquez M. 2014. Approximate decomposition methods for the analysis of multicommodity flow routing in generalized queuing networks. European Journal of Operational Research, 232: 618-629.

[74] Muriel A \& Munsh F. 2004. Capacitated multicommodity network flow problems with piecewise linear concave costs. IIE Transactions, 36: 683-696. 
[75] Murray W \& Shanbhag UV. 2006. A local relaxation approach for the siting of electrical substations. Computational Optimization and Applications, 33: 7-49.

[76] Oudrou A \& MAHeY P. 2000. A minimum-mean cycle cancelling method for multicommodity flow problems. European Journal of Operational Research, 121: 532-548.

[77] Oudrou A, Mahey P \& Vial JP. 2000. A survey of algorithms for convex multicommodity flow problems. Management Science, 46: 126-147.

[78] Paraskevopoulos DC, GÜRel S \& BekTaş T. 2016. The congested multicommodity network design problem. Transportation Research part E, 85: 166-187.

[79] Pardalos PM \& Rosen JB. 1986. Methods for global concave minimization: A bibliographic survey. SIAM Review, 28: 367-379.

[80] Pardalos PM \& VAVASIS S. 1992. Open questions in complexity theory for numerical optimization. Mathematical programming, 57: 337-339.

[81] Resende MGC \& Ribeiro CC. 2003. GRASP with path-relinking for private virtual circuit routing. Networks, 41: 104-114.

[82] Rockafellar RT. 1984. Network Flows and Monotropic Programming, J. Wiley \& Sons.

[83] Saharidis GKD, Minoux M \& Ierapetritou MG. 2010. Accelerating Benders method using covering cut bundle generation. International Transactions in Operational Research, 17: 221-237.

[84] De Souza MC, Mahey P \& Gendron B. 2008. Cycle-based algorithms for multicommodity network flow problems with piecewise convex costs. Networks, 51: 133-141.

[85] Stacey Che, Eyers T \& Anido GJ. 2000. A concave link elimination (CLE) procedure and lower bound for concave topology, capacity and flow assignment network design problems. Telecommunication Systems, 13: 351-372.

[86] Stoer M \& DAhl G. 1994. A polyhedral approach to multicommodity survivable network design. Numerische Mathematik, 68: 149-167.

[87] TARdos E. 1997. Unpublished communication.

[88] TAWARMalani M \& SAHINidis NV. 2005. A polyhedral branch-and-cut approach to global optimization. Mathematical Programming, 103: 225-249.

[89] TUY H. 1964. Concave programming under linear constraints. Soviet Math., 5: 1437-1440.

[90] WARD J. 1999. Minimum aggregate concave cost multicommodity flows in strong series-parallel networks. Mathematics of Operations Research, 24: 106-129.

[91] Weintraub A. 1974. A primal algorithm to solve network flow problems with convex costs. Management Science, 21: 87-97.

[92] Yaged JR B. 1971. Minimum-cost routing for static network models. Networks, 1: 139-172.

[93] ZANGWILL WI. 1968. Minimum concave cost network flows in certain networks. Management Science, 14: 429-450. 